\title{
OBITUARIO
}

\section{In memoriam Doctor Pedro G. Aguilar Fernández: Noviembre 1926 - octubre 2013}

\author{
In memoriam Doctor Pedro G. Aguilar Fernández: November 1926 - October 2013
}

\section{Leonardo Romero}

Facultad de Ciencias Biológicas, Universidad Nacional Mayor de San Marcos. Ciudad Universitaria, Av. Venezuela $\mathrm{s} / \mathrm{n}$. Apartado postal 110058, Lima 11.

Email: Iromeroc@unmsm.edu.pe

\section{Citación:}

Romero L. 2013. In memoriam Doctor Pedro G. Aguilar Fernández: Noviembre 1926 - octubre 2013. Rev. peru. biol. 20(2): 117 - 120 (Diciembre 2013)

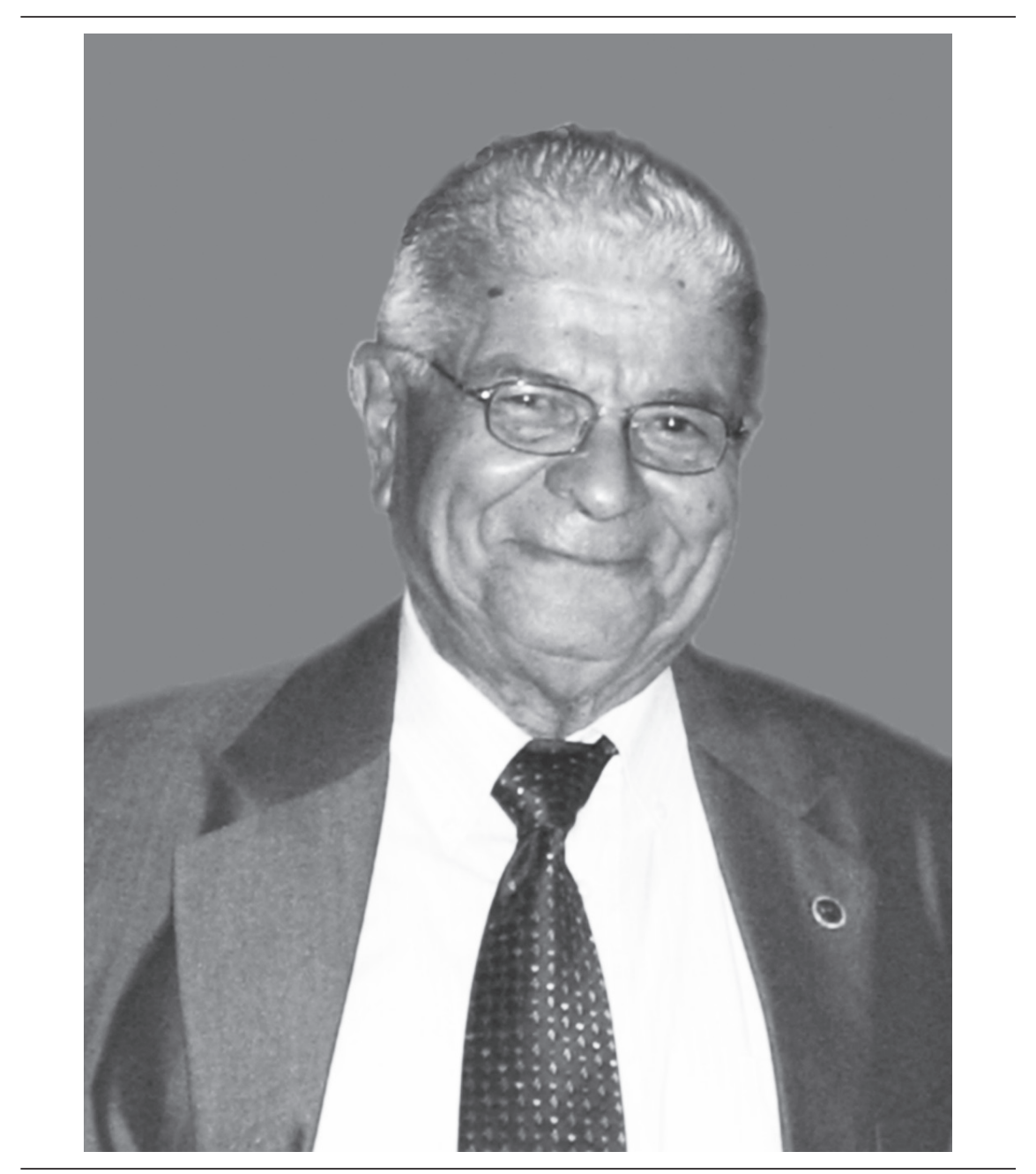

El doctor Pedro Aguilar Fernández falleció el 2 de octubre de 2013. Nació en Cañete, al sur de Lima, el 28 de noviembre de 1926. Colaborador de nuestra revista y colega. Conocido en el ámbito de las ciencias biológicas peruanas, por su participación en diferentes actividades académicas y profesionales. Los académicos e investigadores tendremos muchos motivos para recordar su paso por la vida, encontraremos su presencia en sus trabajos de insectos de las lomas, entomología agrícola, sobre escorpiones, arańas, crustáceos y otros más. Los profesionales biólogos lo recordaremos por su entusiasmo en darle vida a esta labor, fue Pedro Aguilar uno de los miembros fundadores del Colegio de Biólogos del Perú, y quien propuso el Día del Biólogo

○ Los autores. Publicado por la Revista Peruana de Biología de la Facultad de Ciencias Biológicas, Universidad Nacional Mayor de San Marcos. Este es un artículo de acceso abierto, distribuido bajo los términos de la Licencia de Atribución Reconocimiento-NoComercial-SinObraDerivada 3.0 de Creative Commons (http://creativecommons.org/licenses/by-nc-nd/3.0/deed es_ES), que permite el uso no comercial, distribución y reproducción en cualquier medio, siempre que la obra original sea debidamente citadas. Para uso comercial, por favor póngase en contacto con editor.revperubiol@gmail.com. 
“...siendo estudiante del penúltimo año, el 27 de noviembre [1947], en la reunión de homenaje y en agradecimiento al Prof. Augusto Weberbauer...", fecha que posteriormente se oficializo con Decreto Ley N ${ }^{\circ}$. 19364, publicado en El Peruano del 18 de abril de 1972, decreto que crea el Colegio de Biólogos del Perú.

Pedro Aguilar obtuvo su bachiller en Ciencias Biológicas de la Universidad Nacional Mayor de San Marcos en 1949 con la tesis: Insectos polinizadores de plantas silvestres y asilvestradas de Lima y alrededores, y el grado de Doctor en Ciencias Biológicas en la misma universidad en 1954 con la tesis: Adaptaciones de los artrópodos a la vida en las lomas de los alrededores de Lima. Ambas tesis contaron con el patrocinio y orientación de Wolfgang K. Weyrauch.

El doctor Aguilar fue docente en la Universidad Nacional Mayor de San Marcos entre 1959 y 1969. Sin embargo fue en la Universidad Agraria La Molina donde desarrollo más intensamente su labor docente desde 1963 a 1988, llegando a ser Jefe del Departamento de Biología (1970 - 1974). Como profesor se encargó de cursos como: Invertebrados Eucelomados, Entomología, Biología General, Ecología General, Zoología General, Zoología de Invertebrados. A estas labores académicas se sumaban sus labores profesionales de entomólogo agrícola, como Jefe del Servicio Entomológico en el Valle de Lurín (19541959) y en el Valle de Mala (1960-1964).

La formación académica de Pedro Aguilar, su experiencia y su actitud científica lo llevaron al trabajo de editor científico de revistas, libros y otras monografías. Fue el Director-Editor fundador de la Revista Peruana de Entomología (1958 - 1997), la primera revista peruana en el Biological Abstract y que en 1964 recibió de la UNESCO un premio como la mejor revista científica de la región. También fue Director Editor Fundador del Boletín de la Sociedad Entomológica del Perú (1959 - 1973), Director de Anales Cientificos de la UNALM por diez años, miembro del Comité de Redacción del Boletín de Lima por 8 años. Desde 1996 al 2013 fue Editor Científico de las publicaciones seriadas del Instituto del Mar del Perú: el Informe, el Boletin IMARPE, la Memoria Institucional, el Anuario Cientifico Tecnológico y de publicaciones especiales. También fue Editor del Boletín de Investigación del Instituto Tecnológico Pesquero del Perú (2003 - 2010). Tuvo a su cargo la conducción editorial del libro Principios del Control de las Plagas Agricolas de Fausto H. Cisneros (1980, 1ra. edición), del Libro Rojo de la Fauna Silvestre del Perú de Víctor Pulido (1991), del libro Manual de crianza de algunos insectos benéficos a la agricultura peruana de Carlos E. Bartra (1994), del libro Control Químico de Insectos de Fausto H. Cisneros (2012). Además, fue el Editor de libros de resúmenes de diversos congresos de zoología, entomología, ornitología y variados documentos y monografías de la FAO y miembro del Comité Nacional para Edición de Publicaciones Científicas (CONCYTEC, 2002 - 2007).

En honor a su labor académica, el doctor Pedro Aguilar fue nominado Profesor Emérito del Departamento de Biología de la Facultad de Ciencias de la Universidad Nacional Agraria La Molina (27 setiembre de 2002) y Profesor Honorario de la Facultad de Ciencias Biológicas de la Universidad Ricardo Palma (17 agosto de 2007). También, en octubre de 2007 fue reconocido por la Asamblea General Ordinaria de la Sociedad Entomológica del Perú como Co-Presidente Honorario Vitalicio, y por el Colegio de Biólogos del Perú como Socio Fundador, en ocasión del $40^{\circ}$ aniversario de la Ley de Creación del Colegio de Biólogos (18 de abril 2012). Recibió a lo largo de su carrera otras distinciones honoríficas y premios, entre los que podemos resaltar el Premio BAYER $(1978,1995)$ y el Premio WILLE (1989) de la Sociedad Entomológica del Perú, Medalla de Oro Sociedad Entomológica del Perú por 50 ańos de actividad entomológica (1998); Medalla al Mérito Extraordinario por su destacada labor profesional, docente e investigación en entomología del Colegio Nacional de Biólogos del Perú (2002); y el Premio CAMBIE 2003 por Conservación del Ambiente otorgado por la Universidad Científica del Sur.

El doctor Pedro Aguilar fue un caballero, no solamente por su trato cortes, infaltable sonrisa y fuerte apretón de manos al saludarnos, sino por lo que sus obras nos delinean, una persona que sentía un compromiso con la sociedad, con el entomólogo, con el biólogo. Dos de sus publicaciones al respecto están en las páginas de la Revista Peruana de Biología: "El Día del biólogo y su significado en el desarrollo de las ciencias biológicas en el Perú" (Rev. peru. Biol. 2001. 7(2):93-101), y "Comentarios sobre el desarrollo de la Zoología en la Facultad de Ciencias Biológicas de la Universidad Nacional Mayor de San Marcos" (Rev. peru. Biol. 2008. 15 (2):7-14), en ellas podemos leer sobre su visión de los biólogos como constructores de grandes obras, de la integración de sus acciones, la interpretación de sus logros y éxitos, él le proporciona una ilación a la incipiente historia de la biología peruana escrita por los biólogos peruanos. Esto nos permite comprender su interés y dedicación a los grupos donde perteneció académica y profesionalmente.

En el Perú existen pocas revistas científicas, menos aun de ciencias biológicas. Pedro Aguilar tuvo la conducción editorial de notables e importantes revistas científicas, podríamos decir que él fue el primer editor de ciencias biológicas en el Perú. En uno de sus discursos en referencia a Weberbauer él cito a Jorge Basadre:
"Su paciencia de hormiga llevó a Weberbauer a cons- truir fragmento trasfragmento, una obra gigante. Por su cuidado y escrupulosidad en los detalles de ella, parece que hizo suyas las palabras de Goethe: QUE CADA PASO SEA UNA META SIN DEJAR DE SER UN PASO. Resulta pro- fundo por la impotencia para ser superficial. No irradia deslumbradora luz sino precisa claridad. Servidor fiel de la inteligencia, separa lógicamente, distingue, clasifica y luego junta su labor a la que da genial unidad. Es, por encima de los métodos que le corresponden en su propio campo científico, lo contrario de quienes confunden con vehemencia, de quienes citan sin precisión, de quienes se apuran, de quienes se dispersan. En un plano que cabe llamar filosófico, se debe ensalzar su fundamental eficacia. No lo empequeñecen ni siquiera la aspereza polémica o el egoísmo científico. Este hombre menudo y tímido, con pocos amigos y discípulos, que muchas veces conoció la soledad y a veces el hambre, realizó una de las contribuciones más formidables en el Perú del siglo XX."[p:3016, Historia de la República del Perú, 1822 - 1933, Tomo 15, Editada por la Empresa Editora El Comercio S. A. Lima, 2005]

Podríamos vislumbrar en estas palabras que dedica Basadre a Weberbauer, lo que Aguilar adoptaría como el paradigma para él y otros biólogos. Podemos separar en este párrafo directrices para ser escrupulosos en el detalle, sin dejar de saber que aún no 
acaba la obra y que siempre debemos continuarla, ser precisos, profundos, eficaces, ignorar las mezquindades y el egoísmo y sobre todo no olvidar que estamos construyendo una obra formidable. La obra del doctor Pedro Aguilar nos quiso decir eso.

Esta pequeńa nota no hubiera podido ser escrita sin la ayuda de Emira Antonietti del Instituto del Mar del Perú, a quien le quedo muy agradecido.

\section{Publicaciones científicas del doctor Pedro Aguilar}

2001. BENAMÚ M A, AGUILAR P G. Araneofauna presente en huertos de manzano del Valle de Mala, Lima, Perú. Ent. 42:199-210.

1998. TARAZONA J, AGUILAR P G, TOVAR O, ORTEGA H, LAMAS G. Estado del conocimiento de la diversidad biológica en el Perú: una sinopsis. 85-102. En: G. HALFFTER (compilador). La diversidad Biológica en Iberoamerica III. Volumen especial Acta zoológica Mexicana, nueva serie, 223 pp. Instituto de Ecología, Xalapa, México.

1998. AGUILAR P G, RAVEN K G, LAMAS G, REDOLFI I. Conocimiento actual de la diversidad de los insectos registrados para el Perú. 143150 En: G. HALFFTER (compilador). La diversidad biológica en Iberoamerica III. Volumen especial Acta Zoológica Mexicana, nueva serie, 223 pp. Instituto de Ecología, A. C., Xalapa, México.

1995. AGUILAR P G, RAVEN K G, LAMAS G, REDOLFI I. Sinopsis de los hexápodos conocidos del Perú. Rev. per. Ent. 37:1-9.

1994. AGUILAR P. Características faunísticas del norte del Perú. Arnaldoa 2 (1):77-102. Univ. Antenor Orrego, Trujillo.

1992. AGUILAR P. Las Ciencias Biológicas en el Desarrollo del País. En: CASTILLO DE MARUENDA (ed.): Memoria X Congreso Nacional de Biología 02-07 agosto 1992, Lima. Colegio de Biólogos del Perú (354 pp):151-157.

1991. AGUILAR P G. Plagas de las plantas tuberosas tropicales. Manual de manejo integrado. FAO/RLAC. Santiago, Chile. 104 pp.

1990. AGUILAR P G. Sinopsis sobre los eventos del fenómeno "El Niño" en el Perú. Boletín de Lima. 12(70): 69-84.

1989. AGUILAR P G. Ecología. En: Programa de especialización geográfica. 1er. ciclo Curso Intensivo de Geografía del Perú. Febrero 1989. Sociedad Geográfica de Lima. 20 pp.

1988. AGUILAR P G. Las arañas como controladores biológicos de plagas en la agricultura peruana. Rev. per. Ent. 31:1-8.

1987. CALDERON S, AGUILAR P G. Efecto del veneno de Brachistosternus ehrenbergi sobre ratones albinos. Rev. per. Ent. 30: 91-93.

1986. AGUILAR P G, PACHECO V, SILVA T. Fauna desértico costera peruana VIII: Arañas de las Lomas Zapallal, Lima (nota preliminar). Rev. per. Ent. 29: 99-103.

1985. AGUILAR P G, MENDEZ M. Halobates (Hemipt.: Gerridae) en el Océano Pacífico frente al Perú. Rev. per. Ent. 28: 85-88.

1985. AGUILAR P G, UYEYAMA F, AGUILAR Z P. Los "palitos vivientes de Lima" III: un Hydrometridae de las lomas costeras. Rev. per. Ent.28: 89-92.

1985. AGUILAR P G. La Ecología: una ciencia biológica y una breve referencia al Perú. Boletín de Lima. 7 (40): 69-74.

1985. AGUILAR P G. Fauna de las lomas costeras del Perú. Boletín de Lima. 7 (41): $17-28$.

1983. AGUILAR P G. Hechos sobresalientes en el control biológico de las plagas agrícolas en el Perú. En: Informe Final IX Congreso Latinoamericano Zoología: 175-180.

1983. AGUILAR P G. Especies de proscópidos registrados para el Perú. Rev. per. Ent. 26: 85-88.

1981. AGUILAR P G. Apreciaciones sobre la diversidad de los invertebrados en la costa central. Rev. per. Ent. 24: 127-132.

1980. AGUILAR P G. Apuntes sobre control biológico y el control integrado de las plagas agrícolas en el Perú. Rev. per. Ent. 23: 83-110. Con especial colaboración de J M LAMAS, J M SALAZAR, M J DELGADO, E. NÚNEEZ, CH KORYTKOWSKI.

1980. PITTA L, DE PITTA G, AGUILAR P G. Nota sobre los hemocitos del grillo común de Lima (Gryllus peruviensis Saussure) Rev.per. Ent. 23:159-160

1979. AGUILAR P G. Artrópodos epígeos del campo cultivado. I: Estudio preliminar en el algodonero de Cañete. Rev. per. Ent. 22; 87-90
1979. PULIDO V, AGUILAR P. Artrópodos presentes en la dieta de la "lechuza de los arenales" en las lomas de Lachay. Rev. per. Ent. 22: 91-94.

1979. AGUILAR P G. Arañas del campo cultivado VI: Observaciones en algodonales de la costa norte del Perú. Rev. per. Ent. 22:71-73

1979. AGUILAR P G. Arañas del campo cultivado. IV: población de araneidos del algodonero en Chillón y Chancay-Huaral. Rev. per. Ent. 21: 39-41.

1978. AGUILAR P G, GÜEROVICH M. Arañas del campo cultivado. V: Frecuencia de araneidos en algodonales de Mala, Asia y Chilca. Rev. per. Ent. 21: $42-46$.

1977. AGUILAR P, BEINGOLEA O, BRACK A, CEBALLOS I. Vertebrados importantes en la agricultura peruana. Rev. per. Ent. 20; 25-32.

1977. AGUILAR P G. Las arańas en el agroecosistema algodonero de la costa peruana. Anales Científicos. UNAXV(1-4):109-121.

1977. ALEGRE B, MENESES O, AGUILAR P. Peligrosidad de diez especies de arańas comunes en la costa central peruana. Rev. per. Ent. 20: 63-66. 1977.

1977. AGUILAR P G, TÜRKOWSKY J. Fauna desértico-costera peruana. III: Observaciones en el tillandsial de Cajamarquilla, Lima. Rev. per. Ent. 20: 81-85.

1977. AGUILAR P. Fauna desértico costera peruana. IV. Artrópodos del tillandsial de Punta Hermosa. Rev. per. Ent. 20:87-92.

1976. AGUILAR P. Fauna desértico costera peruana-I: Invertebrados más frecuentes en las lomas. Rev. per. Ent. 19:67-70.

1976. AGUILAR P. Arañas del campo cultivado- III: Araneidos en algodonales del valle de Lurín. Rev. per. Ent. 19:71-72.

1976. DÁVILA M, USCA J, AGUILAR P. Estudio comparativo de ocho modelos de trampas para polen. Rev. per. Ent. 19:60-66.

1975. SÁNCHEZ G, AGUILAR P. Notas sobre crustáceos del mar peruano. I: Desarrollo larvario de Lepidopa chilensis Lenz (Decapoda, Anomura: Almuneidae). An. Cient. Univ. Nac. Agraria 13(1-2):1-11.

1975. MÉNDEZ M, SÁNCHEZ G, AGUILAR P. Notas sobre crustáceos del mar peruano. II: Gnathophausia gracilis W. Schum, 1875 (Mysidacea: Lophograstridae). III Eryoneicus sp. (Decapoda, Macrura, Polychelidae). An. Cient. Univ. Nac. Agraria 12 (1-2): 13-18.

A1975. AGUILAR P. Addenda a los Moluscos de las Lomas Costeras del Perú. An. Cient. Univ. Nac. Agraria. 12 (1-2):19.

1975. ARRARTE J, BELLIDO T, AGUILAR P. Moluscos dulceacuícolas del valle del Rímac. An. Cient. Univ. Nac. Agraria 12(3-4):19-26.

1975. AGUILAR P. Arañas del campo cultivado. II. Fluctuación de las familias de araneidos en algodonales de la costa central. Rev. per. Ent. 18:25-28.

1975. AGUILAR P. Nota sobre los Embioptera (Insecta) de la región desértico costera el Perú. Rev. per. Ent. 18:127-128.

1974. AGUILAR P. Apreciaciones sobre la vegetación y flora de las Lomas del Morro Solar, Tablada de Lurín y Atocongo. An. Cient. Univ. Nac. Agraria. 12(1-2):79-84.

1974. AGUILAR P, ARRARTE J. Moluscos de las lomas costeras del Perú. An. Cient. Univ. Nac. Agraria 12 (3-4):93-98.

1974. AGUILAR P. Los palitos vivientes de Lima. II: Proscopidae de las lomas. Rev. per. Ent. 17:107-113

1974. AGUILAR P. Arañas del campo cultivado. I: Población de araneidos en algodonales de Cañete, Rímac y Huaura. Rev. per. Ent. 17:21-27.

1973. AGUILAR P. Especies de grillos registrados para el Perú. Rev. per. Ent. 16:121-123

1973. ARBOLEDA G, MENESES O, AGUILAR P. Escorpiones y escorpionismo en el Perú. III: El veneno del "escorpión de Lambayeque". Rev. per. Ent. 16:78-82

1973. MERINO E, AGUILAR P. Estudios sobre Rogas gossypii Muesebeck, 1960. II: Aspectos de biología y ecología en la costa central. Rev. per. Ent. 16:67-77.

1973. MERINO E, AGUILAR P. Estudios sobre Rogas gossypii Muesebeck, 1960. I: Un método para su crianza masal. Rev. per. Ent. 16:58-66.

1973. AGUILAR P. Apuntes sobre las lomas costaneras del Perú. Bol. Soc. Ent. Perú 7:18-22.

1972. CÁCERES I, AGUILAR P, MENESES O. Escorpiones y escorpionismo en el Perú. II: Efecto del veneno del escorpión de los pedregales" en la costa central. Rev. per. Ent. 15: 38-43.

1972. ESQUERRE DE RAMIREZ A M, MENESES O, AGUILAR P. Acción del veneno de Latrodectus mactans (Fab.) 1775 (Araneida: Theridiidae) sobre ratones, cobayos y perros. Rev. per. Ent. 15:31-37. 
1971. AGUILAR P, MÉNDEZ M. La "arańa chata del nido de arena" Sicarius peruensis (Keyserling), 1880. I: Características morfológicas y ecológicas. Rev. per. Ent. 14(1):143-156

1971. MÉNDEZ M, AGUILAR P. La "araña chata del nido de arena" Sicarius peruensis (Keyserling), 1880. II: Construcción de los nidos. Rev. per. Ent. 14(2):201-212

1971. MÉNDEZ M, AGUILAR P. La "araña chata del nido de arena" Sicarius peruensis (Keyserling), 1880. III: Huevos y desarrollo postembrionario. Rev. per. Ent. 14(2):213-219.

1970. AGUILAR P. Los "palitos vivientes de Lima". I: Los Phasmatidae de las lomas. Rev. per. Ent. 13:1-8

1970. AGUILAR P. Apreciaciones sobre Carpocapsa pomonella L. en el valle de Mala, durante los años 1954-58. Rev. per. Ent. 13:70-75.

1970. AGUILAR P, SAENZ D. Algunas variaciones morfológicas en el grillo común de la costa central. Rev. per. Ent. 13:76-86.

1969. AGUILAR P, MENESES O. Escorpiones y escorpionismo en el Perú. I. Nota preliminar sobre los Scorpionida peruanos. An. Cient. Univ. Nac. Agr. 7(1-2).

1969. AGUILAR P. Principales pestes del algodonero en Mala, Asia y Chilca, durante los ańos 1959 a 1968. An. Cient. Univ. Nac. Agraria 7(1-2).

1969. AGUILAR P. Sanidad del algodonero en el valle de Mala durante los años 1968 y 1969 . Rev. per. Ent. 12:146-152.

1968. AGUILAR P. Nota sobre los escorpiones de Lima. An. Cient. Univ. Nac. Agr. La Molina. 6(3-4):165-172.

1968. AGUILAR P. Araneida frecuentes en el algodonero. Rev. per. Ent. 11(1):92-95
1968. AGUILAR P. Ritmo diurno de afluencia de insectos polinizadores en Lima. Rev. per. Ent. 11:103-107.

1966. CISNEROS F, AGUILAR P. Un ensayo sobre el control químico del "pulgón lanígero del manzano" Eriosoma lanigerum Hausman. Rev. per. Ent. 9:98-100.

1965. AGUILAR P. Nota sobre las arañas del campo cultivado. Rev. per. Ent. 8:80-83.

1965. AGUILAR P. Algunas consideraciones sobre los insectos polinizadores de los alrededores de Lima. Rev. per. Ent. 8:138-145.

1964. AGUILAR P. Rogas gossypii Muesebeck, 1960 durante la campańa 1963-64 en el valle de Mala. Rev. per. Ent. 7:71-74.

1964. AGUILAR P. Especies de artrópodos registrados en las lomas de los alrededores de Lima. Rev. per. Ent. 7:93-95.

1964. AGUILAR P. Control de la polilla del manzano (Carpocapsa pomonella) en el valle de Mala. Rev. per. Ent. 7:103-105.

1963. AGUILAR P. Los artrópodos de las lomas de los alrededores de Lima. Rev. per. Ent. Agr. 6(1):109-114.

1962. AGUILAR P. Observaciones sobre la polilla del manzano (Carpocapsa pomonella L.) en el valle de Mala. Rev. per. Ent. Agr. 4:101-106.

1961. AGUILAR P. Insectos polinizadores más comunes en Lima y alrededores. Rev. per. Ent. Agr. 4:81-82.

1960. AGUILAR P. La introducción al valle de Lurín de Rogas sp. (Hymenopt.; Braconidae), parásito de Anomis texana Riley (Lepidopt.: Noctuidae). Rev. per. Ent. Agr. 3:23-28.

1960. AGUILAR P. La zona de Mala, Asia y Chilca, nuevo récord de acción parasitaria de la avispa Rogas sp. sobre el gusano de la hoja del algodonero. Rev. per. Ent. Agr. 3:63-65.

1954. BAZAN DE SEGURA C, AGUILAR P. Los nemátodos y las enfermedades radicales del algodonero en el Perú. Boletín Fitosanitario FAO. 3(2):20-21 